\title{
Short-ranged attractive colloids: What is the gel state?
}

\author{
E. Zaccarellia ${ }^{\mathrm{a}}$ F. Sciortino ${ }^{\mathrm{a}}$, S.V. Buldyrev ${ }^{\mathrm{b}}$, and P. Tartaglia ${ }^{\mathrm{a}}$ \\ aDipartimento di Fisica and INFM Center for Statistical Mechanics and Complexity, \\ Università di Roma "La Sapienza", Piazzale Aldo Moro 2, 00185 Roma, Italy \\ ${ }^{\mathrm{b}}$ Center for Polymer Studies and Department of Physics, Boston University, Boston, MA \\ 02215, USA.
}

We evaluate thermodynamic, geometric and dynamic properties of a short-ranged square well binary mixture to provide a coherent picture of this simple, but rich, model for colloidal interactions. In particular, we compare the location, in the temperature-packing fraction plane, of the geometrical percolation locus, the metastable liquid-gas spinodal and the glass transition lines. Such comparison provides evidence that the gel-state can not be related to the attractive glass transition line directly. Indications are given for the possibility of an indirect link between the two, via an arrested phase separation process. We finally discuss the possibility that a spherical short range attraction may not be sufficient to produce an equilibrium cluster phase at low packing fraction and low temperatures.

\section{Introduction}

Colloidal dispersions are a suitable class of matter for many scientific purposes. Indeed, these systems are experimentally accessible with light scattering techniques and microscopy, due the the large length scales and time scales involved. Also, the inter-particle interactions can be tuned almost $a d-h o c$, for example by changing the solvent, grafting the particles or adding polymers in the dispersion. Interaction ranges much shorter than the characteristic ones of molecular or atomic liquids can be produced in colloidal suspensions. The interaction range can be reduced to a few per cent of the colloidal particle diameter. Hence, colloids can be used to test a large variety of theoretical models, or vice versa theories can be subjected to stringent experimental tests. Experimentally, the most common realization of short-range attractive colloids is obtained by the addition of small non-adsorbing polymers in the colloidal solution. These, for sufficiently small sizes, can be integrated out of the description[1], and their net effect is to produce an effective attraction on the colloidal spheres, via depletion interactions 2]. The size and the concentration of the polymers control respectively the range and the magnitude of the attraction. Theoretical models of an effective one-component attractive potential are often used, to mimic the experimental situation.

The thermodynamics of short-ranged attractive colloidal systems has been studied in great details 34. By tuning the inter-particle interactions, it has been shown that, for 
spherical hard-sphere colloids, the addition of a particularly narrow range of the attractive part of the potential, with respect to the hard-core diameter, can produce an interesting modification of typical ( $\grave{a}$ la Van der Walls) equilibrium phase diagrams. On decreasing the range of interaction of the attractive potential, the liquid-gas coexistence curve becomes metastable with respect to the crystal, resulting in the disappearance of the liquid as equilibrium phase.

In recent years, the study of the dynamic behaviour of short-ranged attractive colloids has revealed exciting new results. Thanks to theoretical work based on the Mode Coupling Theory (MCT) for the glass transition [5], applied to Baxter sticky spheres [6] and to the short ranged attractive Yukawa model[7], some previous astonishing experimental results 89 , have been revisited and interpreted. MCT theoretical predictions have provided a coherent picture [10 1112 13 14 15] of the dynamic features characteristic of shortranged attractive potentials and have stimulated novel experiments [16 17 18 19 20 21] and simulation studies 22232425262728 . Near the structural arrest dynamics displays far more richness than the one observed in simply repulsive, or long-ranged attractive systems. Many predictions have been confirmed by multiple evidences, some have been questioned, others are still under investigation.

So far, most of the attention of the scientific community has addressed questions regarding the behaviour of short-ranged attractive colloidal systems in the very dense regime of colloidal particles, where the most striking predictions of MCT are manifested. In particular, it is now recognized, with the help of experiments and numerical simulations, that, at high volume fractions, two different mechanisms for glassification exist, one controlled by the excluded volume, commonly referred as 'hard-sphere' (repulsive) glass transition, and one dominated by the attractive interactions, or 'bonding', between particles, commonly termed 'attractive' glass transition. The former glass scenario is only observed at high packing fraction $\phi$, while the latter is manifested for large strength of attraction, i.e. low temperatures or, in the depletion picture, large polymer concentration. These two mechanisms effectively compete with each other [29], when the range of the attractive part of the potential becomes sufficiently short with respect to the hard-core of the particles, giving rise to a reentrant fluid region between the two glasses. The system remains liquid even for packing fractions where a hard sphere repulsive system would be glassy. In MCT, this competition arises from the presence of a higher-order singularity in the control-parameter space [3031, which regulates the anomalous dynamical behaviour in these systems, giving rise to an intriguing logarithmic decay of the density auto-correlation functions, as well as a power-law sub-diffusive behavior for the mean squared displacement. For the square well model, a recent study has provided evidence of the presence of such point 28 .

However, not all predictions share the same robustness with respect to the approximations present in the MCT description. In particular, the theory is ideal, in the sense that it does not take into account the so-called hopping processes, that, as well known, become relevant on approaching the glass transition. These processes allow for residual diffusion where the theory would predict a complete arrest. Indeed, a recent work [27] has investigated the question of the existence of a pure glass-glass transition, as well as the stability of the attractive glass, in relation to activated bond-breaking processes.

Experimentally, dynamical arrest phenomena in short range attractive colloids are observed not only at high density, as discussed above, but also in the low packing fraction 
region. In this case, the arrested material is commonly named a gel. In some cases, the gel phase appears to be contiguous to a cluster phase 3233 34 35, characterized by large supraparticular aggregates, diffusing through the sample in ergodic dynamics. The gel state displays peculiar features like the appearance of a peak in the static structure factor, for very large length scales (of the order of several particle diameters), that is stable in time, as well as a non-ergodic behaviour in the density correlation functions and a finite shear modulus. These solid-like, disordered, arrested features have induced to the appealing conjecture that these colloidal gels can be viewed as the low-density expression of the attractive glasses, both being driven by the same underlying mechanism of arrest. Support to these ideas was found in MCT itself. Indeed, the theory predicts that the attractive glass line extends, practically flat, toward very low packing fractions, almost touching at the critical point the spinodal curve, and following it on its left-hand side. The predicted large values of the non ergodicity parameter along the attractive glass line, similar to the ones observed in the gel phase, and the possibility of modeling the ergodic to non-ergodic transition locus with the attractive MCT line provide support for such an identification [736. Still, some inconsistencies in the interpretation of the gel state as attractive glass have been noted. For example, in the low packing fraction regime, an identification of the attractive glass line with the gel line theory would predict a gel made of particles with average number of bonds less than two [1].

In this paper, we present Molecular Dynamics simulations of a simple model of shortranged potential. In the high packing fraction region, the dynamics of the model displays two different arrest mechanisms, and indeed two different glass transition lines have been located. Here we complement the high density dynamic data with an evaluation of the percolation locus and of the liquid-gas spinodal. We study the intersection between the glass line and the spinodal and show that the former meets the latter at low temperatures, on the high density side. Similarly, no relation is found between the percolation locus and the glass line 37 38]. The outcome of our studies is quite unexpected, and might be crucial for understanding the nature of the colloidal gels.

\section{Model, Theory and Simulation}

We perform Molecular Dynamics simulations of a 50\% - 50\% binary mixture of $N=700$ hard spheres of mass $m$, with diameters $\sigma_{A A}$ and $\sigma_{B B}$ and ratio $\sigma_{A A} / \sigma_{B B}=1.2$. The hard core between particles of different type $\sigma_{A B}=0.5\left(\sigma_{A A}+\sigma_{B B}\right)$. The hard core potential is complemented by an attractive square well potential of depth $-u_{0}$, whose well-width $\Delta_{i j}$ is controlled by varying the parameter $\epsilon=\Delta_{i j} /\left(\Delta_{i j}+\sigma_{i j}\right)$, which is the same for any $i, j$. In this model, a 'bond' between two particles is unambiguously defined when their interaction energy is equal to $-u_{0}$. The simulation is based on a standard event-driven algorithm, implemented for particles interacting with SW potentials [39]. Distances are measured in units of $\sigma_{B B}$, while energy and temperature are measured in units of $u_{o}$, i.e. $k_{B}=1$. Time is measured in units of $\sigma_{B B} \cdot\left(m / u_{0}\right)^{1 / 2}$, mass in units of $m$.

For the particular case $\epsilon=0.03$, results of extensive simulations for this model have been reported in the high density region, in order to make comparison with the existing MCT predictions. This was carried forward in a recent attempt to build an effective mapping between theory and simulation, in order to localize and probe the dynamics 
near the so-called higher order singularity 28].

Ideal glass lines are predicted by MCT as ergodic to non-ergodic transitions, at which by definition the self-diffusion coefficient of atoms, or colloidal particles in our case, is equal to zero. In order to build from our simulations something comparable to an ideal glass line, we have calculated iso-diffusivity curves in the $\phi-T$ plane and studied the evolution of these curves with decreasing diffusivity. Interestingly, the shape of the ideal glass line is maintained up to quite large values of the diffusivity, allowing for a straightforward establishment of the re-entrant behaviour of the glass transition.

Combined with the iso-diffusivity curves, we present here the estimation of liquid-gas 'pre-critical' curve. Such curve is defined as the locus in the $\phi-T$ plane of points where the static structure factor $S(q) \sim 1$ at $q=0$ and provides a close estimate to the spinodal line. Indeed, $S(0)=1$ signals the onset of the divergence of the compressibility, i.e. it is a precursor of the phase separation into gas (colloid-poor) and liquid (colloid-rich) phases.

The square well model is also very well suited for defining a percolation threshold. Indeed, as discussed above, the existence of a bond can be defined unambiguously, when the pair interaction energy is $-u_{0}$. We report here the bond percolation line, beyond which space-spanning clusters of bonded particles are present in the system at a given instant. We estimated it by calculating the cluster connectivity. From an operational point of view, we have defined percolating a state point where more than $50 \%$ of the examined independent configurations (over a total of sixty) displayed an infinite cluster, spanning across the simulation box.

\section{The Phase Diagram}

We plot in Fig 1 the ideal MCT glass lines (GL) for the studied binary SW system with $\epsilon=3 \%$ calculated theoretically using the Percus-Yevick (PY) structure factor [28]. We also report the locus of constant diffusivity, evaluated from MD simulation, with $D / D_{0}$ 40. values varying between $5 \cdot 10^{-3}$ and $5 \cdot 10^{-6}$ together with the locus of zero diffusivity (symbols), obtained extrapolating isothermally the $\phi$-dependence of the diffusion coefficient according to power-law 28]. We also show the transformation of the ideal PY MCT glass line according to the bilinear transformation 31 $\phi \rightarrow 1.897 \phi-0.3922$ and $T \rightarrow 0.5882 T-0.225$, already discussed in [28]. The parameters of the transformation are the result of fitting the locus of zero diffusivity with the transformed MCT curves. Indeed, it is typical that both MCT and the PY solution, in a certain sense, 'overestimates' the glass transition, i.e. always predicting it earlier with respect to reality (for example at higher temperatures in super-cooled liquids or at lower packing fraction for hard spheres). However, differently from hard sphere systems, where this discrepancy requires just a shift in the packing fraction, for an attractive system we have to consider a bilinear mapping [41, which considerably affects the attractive branch of the predicted glass line.

Fig 2 shows the 'spinodal' curve, the percolation curve and the locus of constant diffusivity with $D / D_{0}$ values of $5 \cdot 10^{-2}$ and $5 \cdot 10^{-3}$, significantly extending to lower packing fractions the calculations already presented in [24]). From the data shown in Fig 2 several considerations aris:

(i) the intersection between the isodiffusivity curve and the spinodal provides an esti- 
mate of the characteristic times along the spinodal. Dynamics slows down on increasing density. The intersection between the glass line and the spinodal is located on the high density side. Hence, in this model, the attractive glass line cannot be directly associated to physical phenomena taking place at low packing fractions, i.e. to the gel state.

(ii) The fact that the ideal attractive GL meets the spinodal at high densities suggests an indirect possibility for linking the gel state to the attractive glass. Indeed, if we call $T_{c g}$ the temperature at which the attractive GL meets the coexistence line in the high density side 42], then quenches below $T_{c g}$ may generate upon decomposition regions where the particle concentration is within the attractive glass phase and hence which could arrest kinetically the phase separation process, leaving the imprinting of the phase separation in the frozen structure factor of the system 4344454635 . Such hypothesis, discussed in more details in the next section, although stimulating, unfortunately cannot explain the existence of a contiguity between an "equilibrium" cluster phase and the colloidal gel[32 333 34 35 3.

(iii) The static percolation line is found to start from the low density side of the spinodal, and at all studied temperatures, it remains well to the left side of the largest drawn isodiffusivity curve. This means that the percolating clusters are made of particles which are moving fast, thus the lifetime of the bonds of which the clusters are made at the percolation threshold is extremely short. A study of the lifetime of the infinite cluster [47, which may provide more precise indication of the time stability of the percolating cluster as compared to diffusional times is underway. Still, the short lifetime of the bonds and the extremely large diffusional times suggest that it is not possible to establish any connection between percolation and formation of stable aggregates. On the contrary, we can rule out the possibility that, for the short ranged $\epsilon=3 \%$ model studied here, bond percolation is connected to gelation.

Recently, Miller and Frenkel [48] evaluated the critical point, the spinodal line and the percolation locus for the sticky spheres Baxter model. The Baxter model is the limiting case of the square well model when the width of the well goes to zero and $u_{0}$ goes to infinity. The relation between the sticky parameter $\tau$ [49] and the square well parameters, i.e. $u_{0} / k_{B} T=\log \left(1+1 /\left(4 \tau\left(1 /(1-\epsilon)^{3}-1\right)\right)\right)$, based on the equality of the second virial coefficients for the two models [50], and between the Baxter packing fraction $\phi_{B}$ and the square well one, i.e. $\phi=\phi_{B}(1-\epsilon)^{3}$, allow us to compare the phase diagram of the two systems. Miller and Frenkel [48] data, upon appropriate scaling of the variables, are also reported in Fig 2. The agreement between the pre-critical curve and Baxter spinodal allows us to estimate the critical point for the attractive well to be approximately around $\phi_{c} \sim 0.24, T_{c} / u_{0} \sim 0.31$ for $\epsilon=3 \%$.

\section{Phase Separation and Gels}

We now examine the possibility discussed in the previous section that the gel state is an arrested phase separated system, where the high density phase concentration is in the glass phase. We perform such a study by quenching from $T / u_{0}=1.0$ within the liquid-gas unstable region. We focus for simplicity on a one-component system but, since now we are interested in an aggregation process which involves clustering of many particles, we 


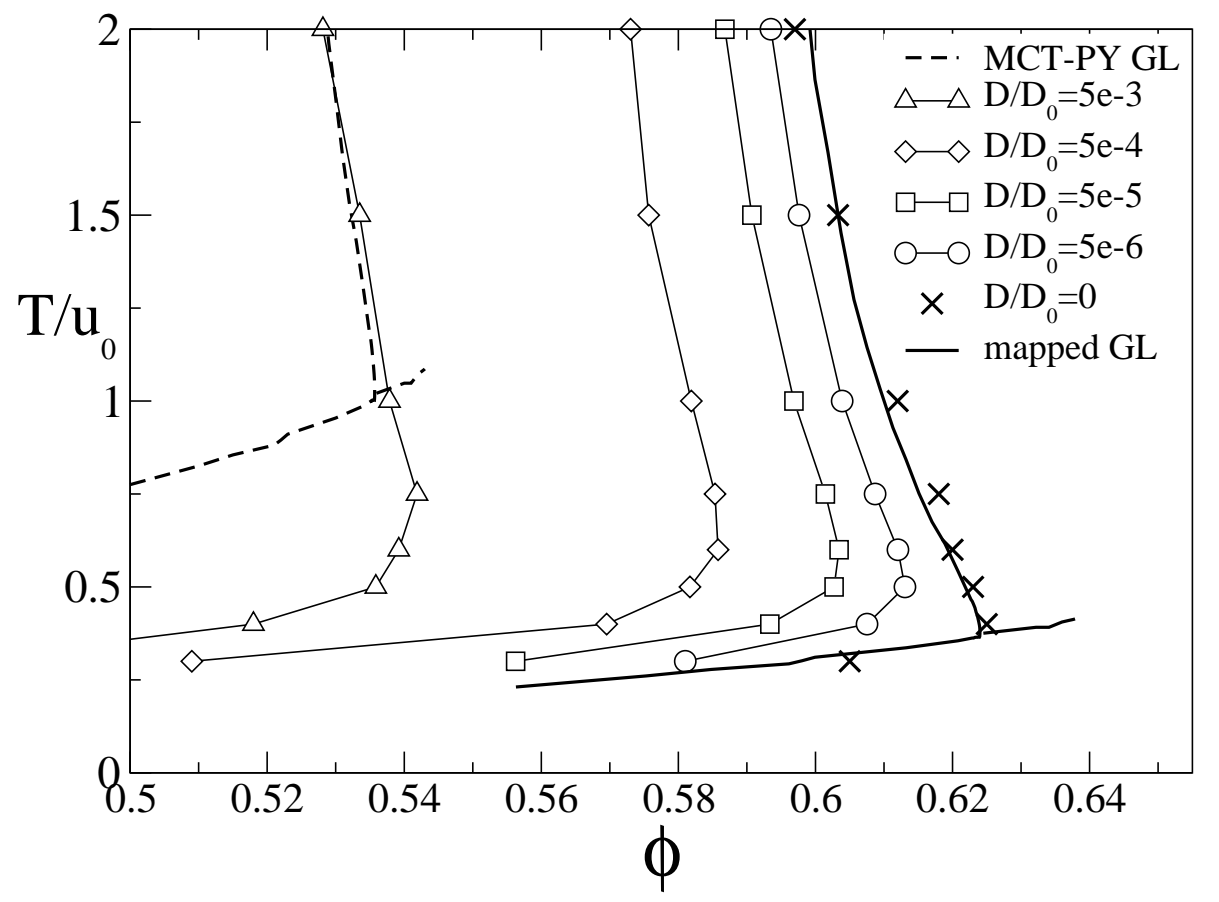

Figure 1. $\left(\phi, T / u_{0}\right)$-diagram in the large colloidal volume fraction region for the binary SW system with $\epsilon=3 \%$. From left to right the reported curves are: the calculated MCT glass line (GL) within Percus-Yevick approximation, iso-diffusivity curves with $D / D_{0}$ equal to $5 \cdot 10^{-3}, 5 \cdot 10^{-4}, 5 \cdot 10^{-5}$ and $5 \cdot 10^{-6}$, power-law extrapolated MD data from Ref. 24] for $D / D_{0}=0$ (crosses), and the MCT GL mapped as shown in Ref. [28. 


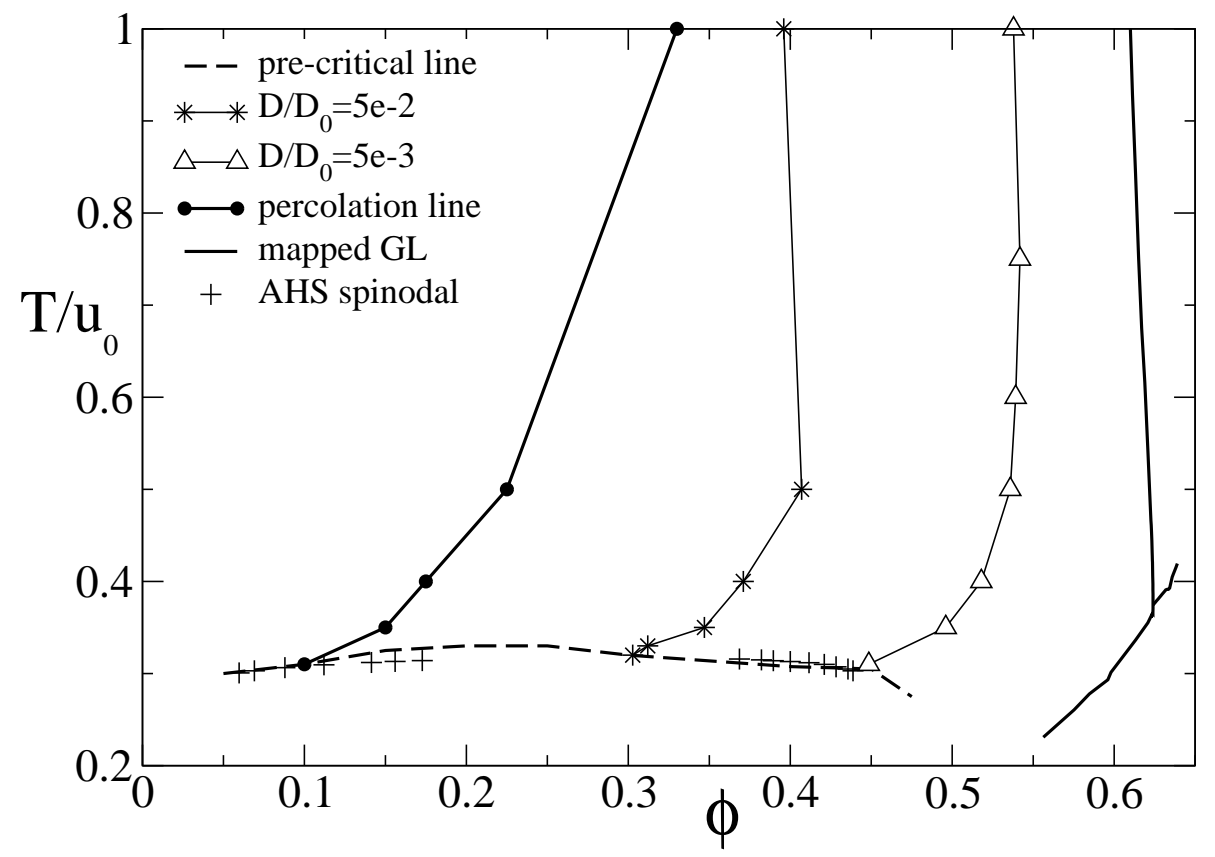

Figure 2. Same as Fig. 1 1 , but reporting the full colloid density region. Together with the mapped GL, already shown in the previous figure, the calculated percolation and precritical lines are reported, as well iso-diffusivity curves for the values $D / D_{0}=5$. $10^{-2}, 5 \cdot 10^{-3}$, both crossing the spinodal line at finite temperature. From these data, one could extrapolate the temperature where the GL meets the spinodal $T_{c g}$ (see text). Also shown for comparison are spinodal points calculated for the Adhesive Hard Sphere (AHS) model, redrawn from [48]. 
consider a much larger system than the one studied previously. We study $N=30000$ particles, interacting via SW potential, with varying well-width, respectively equal to $\epsilon=0.04,0.01,0.005$.

We focus on the low packing fraction $\phi=0.15$, and decrease the temperature. The choice of parameters was made in order to compare our simulation data with the phase diagram reported in 33. Up to approximately $T / u_{0}=0.3$, the system remains in equilibrium, and the static structure factor is similar to that of a normal liquid. However, as we go further in lowering the temperature, i.e. quenching the system to $T / u_{0}=0.2$ and $T / u_{0}=0.1$, we enter in the spinodal regime and phase separation takes place. This causes particles to aggregate, producing a low wavevector peak in the static structure factor, similar, in localization and amplitude, to the one observed in 32. However, differently from what reported in those experiments, the structure factor continues to evolve, during the simulation, although on logarithmic time-scales. This phenomenon is more marked for the largest studied well-width, i.e. $\epsilon=0.04$, and for the highest temperature considered for the smaller widhts, $T / u_{0}=0.2$.

In Figure 3- (upper panel), we report the time evolution of the energy per particle $U / N$ for the various studied cases. It can be observed that there is a characteristic time-scale, which controls the aggregation kinetics. After the microscopic time-scale, there is a strong decrease in the energy, which then crosses over to a regime of very slow, approximately logarithmic, decay. The decay is slower, the narrower the studied well-width and the lower the temperature. We note that the number of bonds per particle tends to a value of about 3 or more, which, taking into account the low packing fraction of the system, indicates the formation of a cluster network. The resulting cluster phase is not an equilibrium phase, in the sense that it is driven by spinodal decomposition. To support such interpretation we show in Figure 3- (lower panel), the evolution of the static structure factor for the case $\epsilon=0.01$ and $T / u_{0}=0.1$. The various curves represents $S(q)$ at different times from the quench, which are logarithmically spaced between $t_{1} \sim 10$ and $t_{2} \sim 10^{4}$. Times shorter than thermal equlibration are not reported. To better quantify the time dependence of the separation process, we report in Figure 4 the time evolution of the peak intensity $S_{M A X}$ (upper panel), in analogy with the inset of Fig. 1 in Ref. 32. For the larger well-width $\epsilon=0.04$, the increase of the amplitude in $S(q)$, although on logarithmic time-scale, is still clearly observable after two decades in time [51. However, for the two very narrow widths $1 \%, 0.5 \%$ at the extremely low temperature $T / u_{0}=0.10$ a significantly flatter behaviour is observed. Indeed, the two curves are almost superimposed onto each other at long times, and the narrower well-width shows a sharper crossover to the quasi-plateau.

Also, in Figure 4- (lower panel), we plot the time evolution of the first moment of $S(q)$, i.e. $q_{1}=\left[\sum_{q} q S(q)\right] /\left[\sum_{q} S(q)\right]$, which has a scaling equivalent to the peak position, but which can be calculated much more accurately [52. These curves can be fitted at large times with a power-law $\sim t^{-\beta}$, where $\beta$ varies between 0.25 and 0.5 , depending on the case and on the range of fit. We recall that the typical exponent for spinodal decomposition is $1 / d, d$ being the dimensionality [5345]. This result is consistent with the typical scaling of spinodal decomposition, and provides another indication that the aggregation we observe is driven by the gas-liquid phase transition. The two cases of extremely short-ranged and strong attraction display an extremely slow dynamics, which almost arrest at large times. If we return to the situation examined in the previous paragraph, we know that 

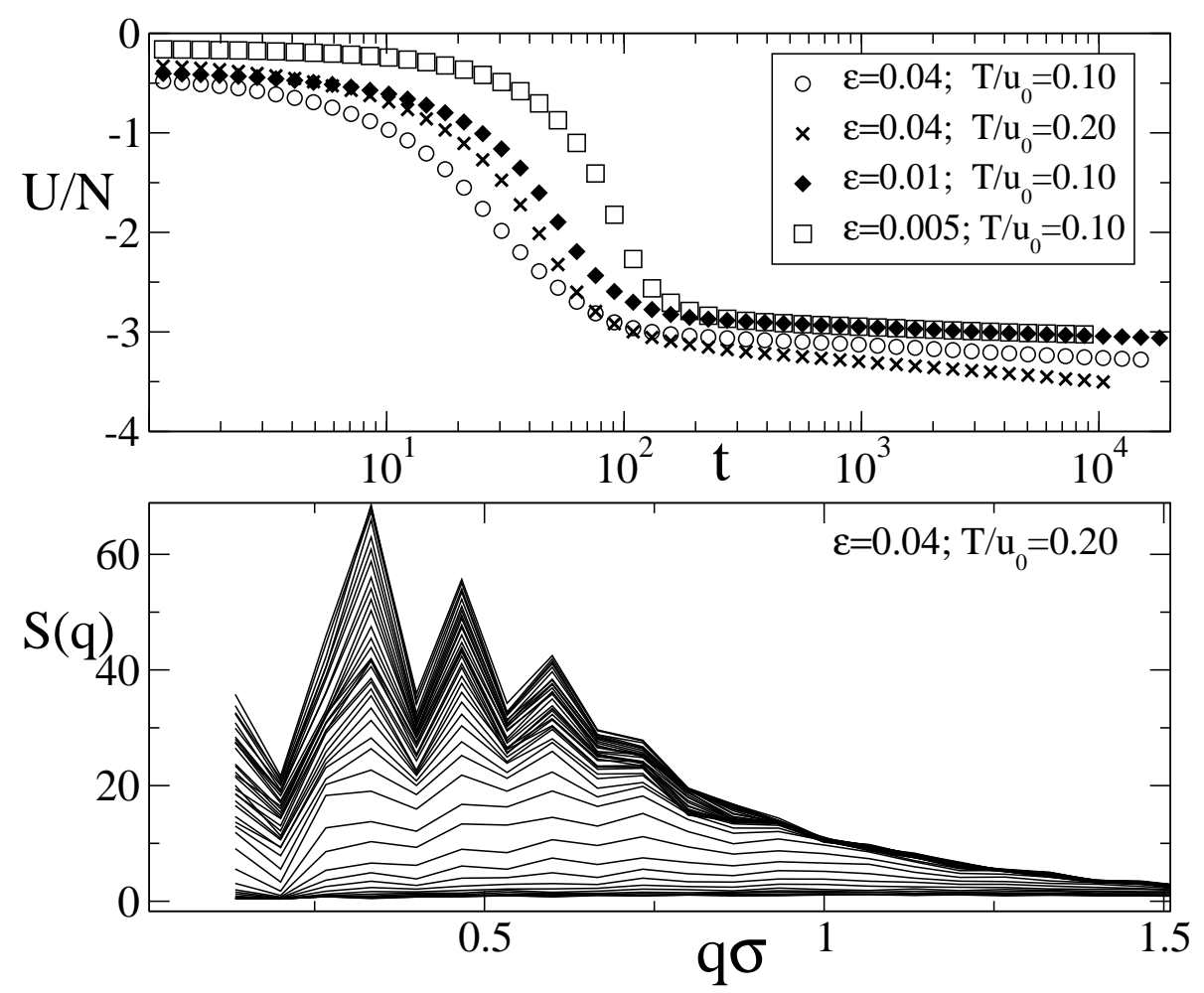

Figure 3. Upper Panel: Time evolution of the energy per particle for a one-component SW model at $\phi=0.15$, after the quenches to $T / u_{0}=0.2$ and $T / u_{0}=0.1$. Various wellwidths; Lower Panel: Static structure factors after the quench for the case $\epsilon=0.04$ and $T / u_{0}=0.2$. The various curves refer to different times, equally spaced on a logarithmic scale. The significant noise is due to the fact that we show a single realization. 


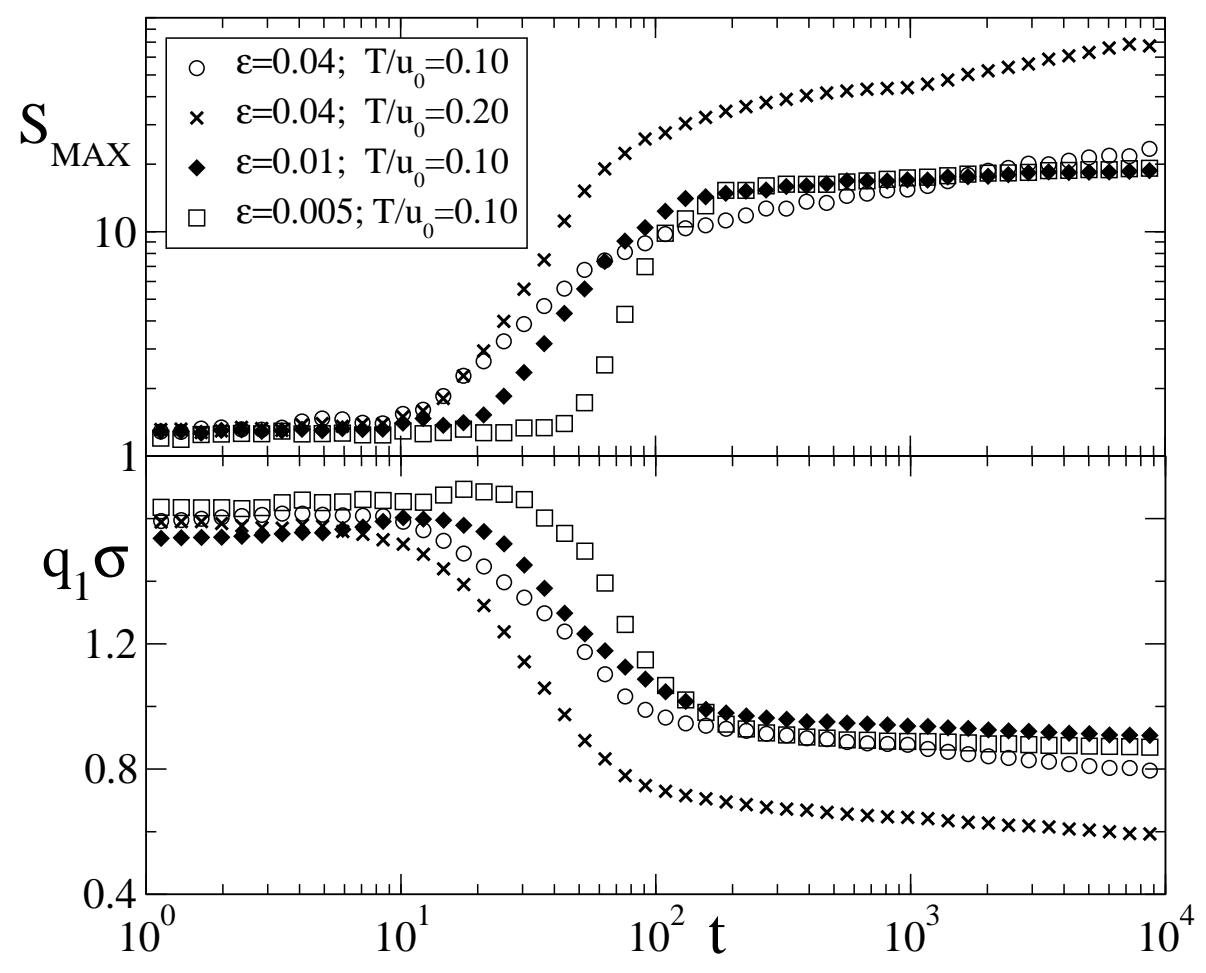

Figure 4. Upper Panel: Time evolution after the quenches of the static structure factor maximum $S_{M A X}$, for the same cases as in Fig. 3 (upper panel); Lower Panel: Time evolution after the quenches of the first moment $q_{1}$ in units of $\sigma$ of the static structure factor. 
the attractive branch of the glass line meets the spinodal, at some very low temperature $T_{c g}$. It might be possible that these two cases already correspond to a temperature lower than $T_{c g}$, thus what we observe here are indirect gels induced by spinodal decomposition.

\section{Conclusions}

In this paper we have provided evidence of two new important facts for simply shortranged attractive potentials, of the kind generated via depletion interactions. Firstly, the slowing down of the dynamics in short-ranged attractive systems truly arises only at very high densities, far away from the percolation line. At low temperatures, the attractive glass line ends in the spinodal on the high density side. Secondly, a gel contiguous to an equilibrium cluster phase does not manifest. A gel could result from an arrested phase separation process when the density of one of the two phases crosses the attractive glass transition at low temperatures and for very short ranges of attraction $\left(T / u_{0}=0.1\right.$, $\epsilon \leq 0.01$ ). At even lower temperatures, diffusion limited cluster aggregation[54] will take over, leaving the imprinting of the aggregation process in the gel structure 5556.

These two observations clash with earlier conjectures that a gel state, considered as a natural extension to lower densities of the ideal MCT attractive glass, would be found in these systems [736. We also note that the data reported in this article appear in disagreement with the recent theoretical model [57] (see in particular Fig. 2 of Ref. [57]), where a gel state is identified with an attractive glass after a renormalization procedure. On the other hand, our results shed some light on the fact that in real systems there must be an additional mechanism that allows to stabilize an equilibrium cluster phase. Evidence is now emerging in the scientific community that colloidal particles often tend to have a residual charge distribution [583459, whose net effect is a weak, long-ranged repulsive barrier, whose importance in governing the dynamics of the system may manifest when many particles are clustered together, preventing further aggregation 60]. It would be interesting to find out if the stabilizing effect of electro-static forces implies that gels are formed by caging of clusters, instead of by caging of particles. If this were the case, particle gels would be driven by a completely different mechanism than attractive glasses, ultimately being a different form of glasses, where particles are replaced by clusters of many particles, whose size and distribution will depend on the amount of charge that is present in the colloidal suspension. Work is in progress to address specifically such possibilities.

\section{Acknowledgments}

We acknowledge support from MIUR PRIN, FIRB and INFM PRA-GENFDT, and also from INFM Iniziative Calcolo Parallelo. We thank W. C. K. Poon for interesting discussions.

\section{REFERENCES}

1. C. N. Likos, Phys. Rep. 348, 267 (2001).

2. S. Asakura, F. Oosawa, J. Polym. Sci. 33, 183 (1958).

3. D. Frenkel, Science 296, 106 (2002). 
4. V. J. Anderson and H.N.W. Lekkerkerker, Nature 416, 811 (2002).

5. W. Götze, in Liquids, Freezing and Glass Transition, p.287, J.P. Hansen, D. Levesque and J. Zinn-Justin (eds.), North Holland, Amsterdam (1991).

6. L. Fabbian, W. Götze, F. Sciortino, P. Tartaglia and F. Thiery, Phys. Rev. E 59, R1347 (1999) and Phys. Rev. E 60, 2430 (1999).

7. J. Bergenholtz and M. Fuchs, Phys. Rev. E 59, 5706 (1999).

8. P. N. Pusey, A. D. Pirie and W. C. K. Poon, Physica A

9. E. Bartsch, M. Antonietti, W. Schupp, and H. Sillescu, J. Chem. Phys. 97, 3950 (1992).

10. K. A. Dawson, G. Foffi, M. Fuchs, W. Götze, F. Sciortino, M. Sperl, P. Tartaglia, T. Voigtmann, and E.Zaccarelli, Phys. Rev. E 63, 011401 (2001).

11. E. Zaccarelli, G. Foffi, K. A. Dawson, F. Sciortino and P. Tartaglia, Phys. Rev. E 63, 031501 (2001).

12. G. Foffi, G. D. McCullagh, A. Lawlor, E. Zaccarelli, K.A. Dawson, F. Sciortino, P. Tartaglia, D. Pini and G. Stell, Phys. Rev. E 65, 031407 (2002).

13. K. A. Dawson, G. Foffi, G. D. McCullagh, F. Sciortino, P. Tartaglia, E. Zaccarelli, Journ. Phys.: Condens. Matt. 14, 2223 (2002).

14. E. Zaccarelli, F. Sciortino, P. Tartaglia, G. Foffi, G. D. McCullagh, A. Lawlor and K. A. Dawson, Physica A 314/1-4, 539 (2002).

15. W. Götze and M. Sperl, J. Phys. Condens. Matt. 15, S869 2003.

16. F. Mallamace, P. Gambadauro, N. Micali, P. Tartaglia, C. Liao and S. H. Chen, Phys. Rev. Lett. 84, 5431 (2000).

17. K. N. Pham, A. M. Puertas, J. Bergenholtz, S. U. Egelhaaf, A. Moussaïd, P. N. Pusey, A. B. Schofield, M. E. Cates, M. Fuchs, and W. K. Poon, Science 296, 104 (2002).

18. T. Eckert and E. Bartsch, Phys. Rev. Lett. 89, 125701 (2002).

19. W.R. Chen, S.H. Chen and F. Mallamace, Phys. Rev. E, 66021403 (2002); W.R. Chen et al, Phys. Rev. E, 68041402 (2003).

20. S. H. Chen, W. R. Chen and F. Mallamace, Science, 300, 619 (2003).

21. K. N.Pham, S. U.Egelhaaf, P. N.Pusey, and W. C. K. Poon to be published (2003); cond-mat/0308250.

22. A. M. Puertas, M. Fuchs, M. E. Cates, Phys. Rev. Lett. 88, 098301 (2002)

23. G. Foffi , K.A. Dawson, S. Buldyrev, F. Sciortino, E. Zaccarelli and P. Tartaglia, Phys. Rev. E 65, 050802 (2002).

24. E. Zaccarelli, G. Foffi, K. A. Dawson, S. V. Buldyrev, F. Sciortino, P. Tartaglia, Phys. Rev. E 66, 041402 (2002).

25. E. Zaccarelli, G. Foffi, K. A. Dawson, S. V. Buldyrev, F. Sciortino and P. Tartaglia, J. Phys.: Condens. Matter 16,S367 (2003).

26. A. M. Puertas, M. Fuchs, M. E. Cates, Phys. Rev. E 67, 031406 (2003).

27. E. Zaccarelli, G. Foffi, F. Sciortino and P. Tartaglia, Phys. Rev. Lett. 91, 108301 (2003).

28. F. Sciortino, P. Tartaglia, and E. Zaccarelli, submitted (2003); condmat/0304192.

29. F. Sciortino, Nature Materials, News and Views, 1, 145 (2002).

30. W. Götze and M. Sperl, Phys. Rev. E 66, 011405 (2002).

31. M. Sperl, Phys. Rev. E 68,031405 (2003); Phys. Rev. E , in press (2003).

32. P.N. Segrè, V. Prasad, A.B.chofield, and D.A. Weitz, Phys. Rev. Lett. 86, 6042 (2001). 
33. V. Prasad, V.Trappe, A.D.Dinsmore, P.N.Segrè, L. Cipelletti,and D.A. Weitz, Faraday Discuss. 123, 1 (2003).

34. A. D. Dinsmore and D. A. Weitz, J. Phys. Condens. Matt. 14, 7581 (2002).

35. H. Segdwick, K. Kroy, M. B. Robertson, S. U. Egelhaaf and W. C. K. Poon, submitted (2003); condmat/0309616.

36. J. Bergenholtz, M. Fuchs and W. C. K. Poon, Langmuir 19, 4493 (2003).

37. M.C. Grant and W.B. Russel Phys. Rev. E 47, 2606 (1993).

38. S. K. Kumar and J. F. Douglas, Phys. Rev. Lett. 87, 188301 (2001).

39. D. C. Rapaport, The Art of Molecular Dynamic Simulation, Cambridge University Press, 1995.

40. We recall that $D_{0}=\sigma \sqrt{T / m}$, is a normalizing factor, which ensures that the difference in the average velocities due to the temperature is eliminated, so that the diffusion coefficient is comparable between different temperatures.

41. This mapping is based on the hypothesis that the parameter $\epsilon$ does not require any transformation, based on the observation that it is the crucial parameter governing the anomalous behaviour of the system and the existence of the higher order singularity.

42. The ideal GL, being the outcome of the ideal MCT does not coincide with the "calorimetric" glass transition line. While in hard-spheres hopping phenomena are not significant due to the absence of an energy scale, in molecular systems it is well known that the "MCT glass" is destabilized by activated processes, which slowly restore ergodicity. Indeed, in these systems the calorimetric glass transition temperature is located well below the MCT critical temperature. In short-ranged attractive colloids, activated processes can be associated with thermal fluctuations of order $u_{0}$, which are able to break the bonds. These processes generate a finite bond lifetime and destabilize the attractive glass. Hence the effective $T_{c g}$ must lie below the value calculated on the basis of the ideal MCT attractive GL.

43. F. Sciortino, R. Bansil, H. E. Stanley, P. Alstrom, Phys. Rev. E 47, 4615 (1993).

44. D. Sappelt, J. Jackle, Europhys. Lett. 37, 13 (1997).

45. P. Poulin, J. Bibette and D. A. Weitz, Eur. Phys. J. B 7, 277 (1999).

46. W. C. K. Poon, A. D. Pirie and P. N. Pusey, Faraday Discuss. 101, 65 (1995).

47. F. Sciortino, P. H. Poole, H. E. Stanley, and S. Havlin Phys. Rev. Lett. 64, 1686 (1990).

48. M. Miller and D Frenkel, Phys. Rev. Lett. 90, 135702 (2003).

49. R. J. Baxter, J. Chem. Phys. 49, 2770 (1968).

50. M. G. Noro and D. Frenkel, J. Chem. Phys. 113, 2941 (2000).

51. To give an estimation of this time in computer time, these simulations have required approximately 4 months in CPU time.

52. S. C. Glotzer, M. F. Gyure, F. Sciortino, A. Coniglio and H. E. Stanley, Phys. Rev. E 49, 247 (1994).

53. J. M. Gunton, M. San Miguel, P. S. Sahni, in Phase Transition and Critical Phenomena 8, 267, J.L. Lebowitz (ed.), Academic Press, London (1983).

54. For a general review see, e.g., T. Vicsek, Fractal Growth Phenomena, World Scientific, Singapore (1989).

55. M. Carpineti and M. Giglio, Phys. Rev. Lett. 68, 3327 (1992).

56. F. Sciortino and P. Tartaglia, Phys. Rev. Letts. 74, 282 (1995). 
57. K. Kroy, M. E. Cates and W. C. K. Poon, condmat/0310566.

58. A. Yethiraj and A. van Blaaderen, Nature 421, 513 (2003).

59. W. C. K. Poon, private communication.

60. J. Groenewold and W. K. Kegel, J. Phys. Chem. B 105, 11702 (2001). 